ISSN 0975-3303

Mapana J Sci, 11, 4(2012), 99-108

https:/ / doi.org/ 10.12725/ mjs.23.8

\title{
Graphs in Network Flows
}

\author{
V Manjula*
}

\section{Abstract}

This paper presents a collection of basics and application of Network flows in Graph theory which is an out- growth of set of lecture notes on Graph applications. It is not only a record of material from text books but also a reflection of precise graphical concept which will be useful for students where such facts are needed. There are many real life problems dealing with discrete objects and binary relations and graph is very convenient form of its representation. A network flow graph $G=(V, E)$ is a directed graph with two special vertices: the source vertex $\mathrm{s}$, and the sink vertex $\mathrm{t}$. Many problems in the real world are to be solved using maximum flow. "Real" networks, like the Internet or electronic circuit boards, are good examples of flow networks. Generally graphs can be used in two situations. Firstly since graph is a very simple, convenient and natural way of representing the relationship between objects. Secondly we have graph as model solve the appropriate graph theoretic problem then interpret the solution in terms of original problem In the modern world, planning efficient routes is essential for business and industry, The flow of information or water or gas etc in a network are useful to find the max rate of flow that is possible from one station to another A Transport network represents a general model for

* Basic Engineering Department, DVR \& Dr HS MIC College of Technology, Kanchikacherla, Krishna State, Andhra Pradesh 521180 India, manju_adiraju@yahoo.co.in 
transportation of material from origin of supply to destination through shipping routes. The objective of this paper is to discuss the concepts and terminology of Network flows with Graphical representations.

\section{Introduction}

In the modern world, planning efficient routes is essential for business and industry, with applications as varied as product distribution, laying new fiber optic lines for broadband internet, and suggesting new friends within social network websites like Face book etc In graph theory, a flow network is a directed graph where each edge has a capacity and each edge receives a flow. The amount of flow on an edge cannot exceed the capacity of the edge. Often in Operations Research, a directed graph is called a network, the vertices are called nodes and the edges are called arcs. A flow must satisfy the restriction that the amount of flow into a node equals the amount of flow out of it, except when it is a source, which has more outgoing flow, or sink, which has more incoming flow. A network can be used to model traffic in a road system, fluids in pipes, currents in an electrical circuit, or anything similar in which something travels through a network of nodes $[1,2,3,4]$.

\section{Why Study Graphs in OR}

Graph theory is a powerful tool in operations research. A graph is a mathematical abstraction that is useful for solving many kinds of problems. Generally a graph can be used as a model for a network through which some commodity is transported from one place to another. The general problem in such transport network is to maximize the flow or minimize the cost of a prescribed flow. Usually the problem can be solved by linear programming but graph theoretic approach has been found to be computationally more efficient $[5,6]$.

\section{Definitions}

A network is a directed graph, $G=(V, E)$. A capacity function, c : $\mathrm{E} \rightarrow[0, \infty)$, maps each edge to how much "traffic" it can carry. Two vertices in the graph, $\mathrm{s}$ and $\mathrm{t}$, are distinguished as the source 
and sink, respectively. The source vertex has only outgoing edges and the sink has only incoming edges.

Flow. Given a network $G=(V, E)$, a flow is a function $f: E \rightarrow[0, \infty)$ such that:

1. $\forall \mathrm{e} \in \mathrm{E}: \mathrm{f}(\mathrm{e}) \leq \mathrm{c}(\mathrm{e}) 2 . \forall \mathrm{v} \in \mathrm{V} \backslash\{\mathrm{s}, \mathrm{t}\}: \sum(\mathrm{u}, \mathrm{v}) \square \mathrm{E}, \mathrm{f}(\mathrm{u}, \mathrm{v})=\sum(\mathrm{v}$, u) $\square \mathrm{Ef}(\mathrm{v}, \mathrm{u})[7]$.

\section{Interpretation}

Due to technical restrictions some jobs must be finished before others can be started such as washing before drying, putting foundation before erecting walls etc. Each activity also requires a certain time called the duration of the activity. A weighted digraph can be drawn to depict the project such as each edge represents an activity and its weight represents the duration of the activity. The vertices represent beginning and ending of the activities and are called events in the project $[8,9]$.

\section{Activity networks in project planning}

One of the most popular and successful application of network flow is in the planning and scheduling of large complicated projects. The two best methods are CPM-Critical path method and PERT-Program evaluation and review technique.

Critical Path Analysis (CPA) or the Critical Path Method (CPM) helps to plan all tasks that must be completed as part of a project. They act as the basis both for preparation of a schedule, and of resource planning. During management of a project, they help to monitor achievement of project goals. Critical Path Analysis are presented using circle and arrow diagrams.

In these, circles show events within the project, such as the start and finish of tasks An arrow running between two event circles shows the activity needed to complete that task

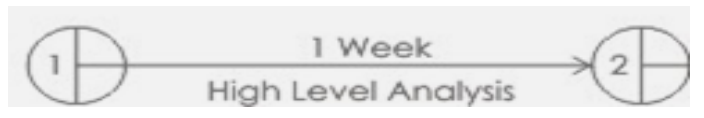


Simple circle and arrow diagram

PERT is a variation on Critical Path Analysis that takes a slightly more skeptical view of time estimates made for each project stage. To use it, estimate the shortest possible time each activity will take, the most likely length of time, and the longest time that might be taken if the activity takes longer than expected.

Use the formula below to calculate the time to use for each project stage:

$$
\text { shortest time }+4 \mathrm{x} \text { likely time }+ \text { longest time }
$$

\section{6}

This helps to bias time estimates away from the unrealistically short time-scales normally assumed [10].

\section{Network Diagram Representation}

In project scheduling the first step is to sketch an arrow diagram which shows inter dependencies and precedence relations among project. Each activity can be represented by one and only one arrow in the network.

A flow network $G=(V, E)$ is a directed graph where each edge $(u$, v) [[proper subset]] E has a capacity $\mathrm{c}(\mathrm{u}, \mathrm{v})>=0$.

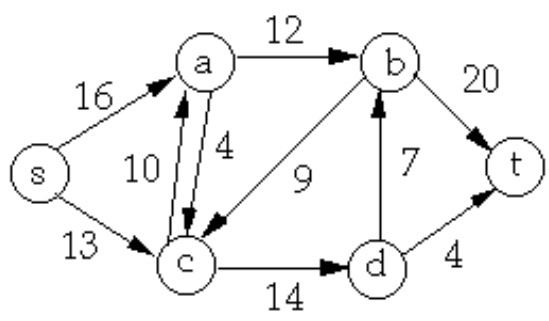

Fig. 1

Source (s) - node with only outflows 
Sink (t) - node with only inflows

Assume graph is connected

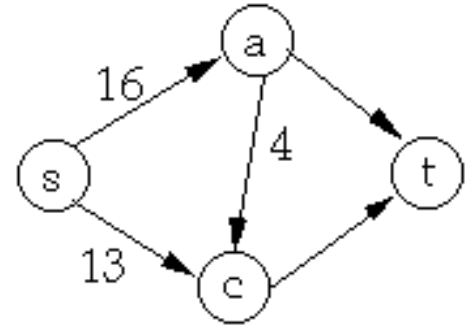

Fig. 2

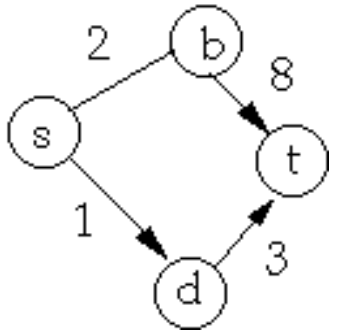

Fig. 3

Application areas of activity network

1. Building constructions

2. Administration

3. Maintenance planning

4. Marketing

5. Research and development

6. Manufacturing

\section{Drawing the CPM/PERT Network}

Each activity (or sub-project) in a PERT/CPM Network is represented by an arrow symbol. Each activity is preceded and succeeded by an event, represented as a circle and numbered [10]. 


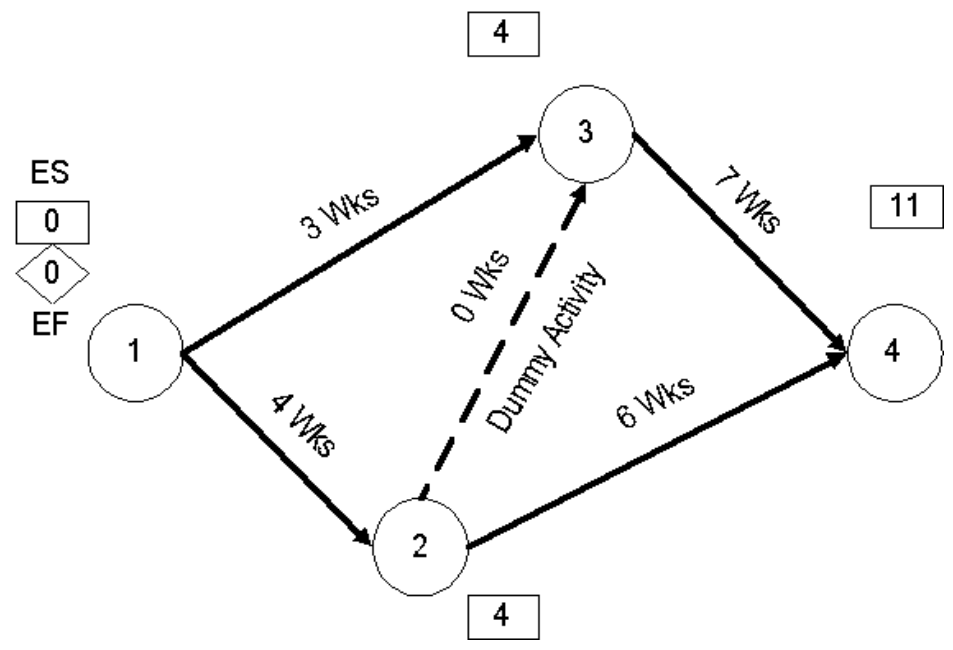

Fig. 4

At Event 3, we have to evaluate two predecessor activities Activity 1-3 and Activity 2-3, both of which are predecessor activities. Activity 1-3 gives us an Earliest Start of 3 weeks at Event 3. However, Activity 2-3 also has to be completed before Event 3 can begin. Along this route, the Earliest Start would be $4+0=4$. The rule is to take the longer (bigger) of the two Earliest Starts. So the Earliest Start at event 3 is 4.Similarly, at Event 4, we find we have to evaluate two predecessor activities - Activity 2-4 and Activity 3-4. Along Activity 2-4, the Earliest Start at Event 4 would be 10 wks, but along Activity 3-4, the Earliest Start at Event 4 would be 11 wks. Since 11 wks is larger than 10 wks, we select it as the Earliest Start at Event 4.We have now found the longest path through the network. It will take 11 weeks along activities 1-2, 2-3 and 3-4. This is the Critical Path. Bipartite Matching: A matching is a subset of the edges $M \subseteq E$ such that for all $v$ \&in; $V$, zero or one edges of $M$ are incident on $v$. (1: $v$ is matched; $0: v$ is unmatched.)A typical assignment problem, presented in the classic manner, is shown in Fig. Here there are five machines to be assigned to five jobs. The numbers in the matrix indicate the cost of doing each job with each machine. Jobs with costs of $\mathrm{M}$ are disallowed assignments. The problem is to find the minimum cost matching of machines to jobs. 


\begin{tabular}{c|c|c|c|c|c|}
\multicolumn{1}{c}{} & \multicolumn{1}{c}{$\mathrm{J} 1$} & \multicolumn{1}{c}{$\mathrm{J} 2$} & \multicolumn{1}{c|}{$\mathrm{J} 3$} & \multicolumn{1}{c|}{$\mathrm{J} 5$} \\
\cline { 2 - 6 } M1 & $\mathrm{M}$ & 8 & 6 & 12 & 1 \\
\cline { 2 - 6 } M2 & 15 & 12 & 7 & $\mathrm{M}$ & 10 \\
\cline { 2 - 6 } M3 & 10 & $\mathrm{M}$ & 5 & 14 & $\mathrm{M}$ \\
\cline { 2 - 6 } M4 & 12 & $\mathrm{M}$ & 12 & 16 & 15 \\
\cline { 2 - 6 } M5 & 18 & 17 & 14 & $\mathrm{M}$ & 13 \\
\cline { 2 - 5 } & & &
\end{tabular}

Matrix model of the assignment problem.

The network model is in Fig. It is very similar to the transportation model except the external flows are all +1 or -1 . The only relevant parameter for the assignment model is arc cost all other parameters should be set to default values. The assignment network also has the bipartite structure.

\section{The Max-flow Min-cut Theorem}

Let $f$ be a flow in $G=(V, E)$ be a flow network with sources and sink.

The following are equivalent:

1. $\mathrm{f}$ is a maximum flow in $\mathrm{G}$

2. The residual network Gf contains no augmenting paths

3. $|\mathrm{f}|=\mathrm{c}(\mathrm{S}, \mathrm{T})$ for some cut $(\mathrm{S}, \mathrm{T})$ of $\mathrm{G}[12]$ :

Let $G=(V, E)$ be a flow network and $f$ a flow in $G$ A cut $(S, T)$ of $G$ is a partition of $\mathrm{V}$ into $\mathrm{S}$ and $\mathrm{T}=\mathrm{V}-\mathrm{S}$ such that :s [[proper subset]] $S$ and $t[$ proper subset]] $T$.

The net flow across the cut $(S, T)$ is $f(S, T)$.

The capacity of the cut $(S, T)$ is $c(S, T)[13]$. 
V Manjula

ISSN 0975-3303

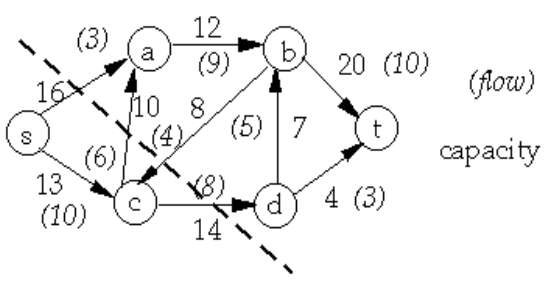

Fig. 5

$S=\{s, c\} T=\{a, b, d, t\}$

$f(S, T)=3+6-4+8=13$

$c(S, T)=16+10+14$

Let $\mathrm{G}=(V, \mathrm{E})$ be a flow network and $\mathrm{f}$ be a flow in $\mathrm{G}$.

Let $\mathrm{Gf}=(\mathrm{V}, \mathrm{Ef})$ be the residual network of $\mathrm{G}$ induced by $\mathrm{f}$.

An augmenting path $\mathrm{p}$ is a simple path from $\mathrm{s}$ to $\mathrm{t}$ in $\mathrm{Gf}$

Residual capacity of $p$ is the maximum amount of net flow that we can ship along the edges of $p$,

$$
\operatorname{cf}(p)=\min \{c f(u, v):(u, v) \text { is on path } p\}[13]
$$

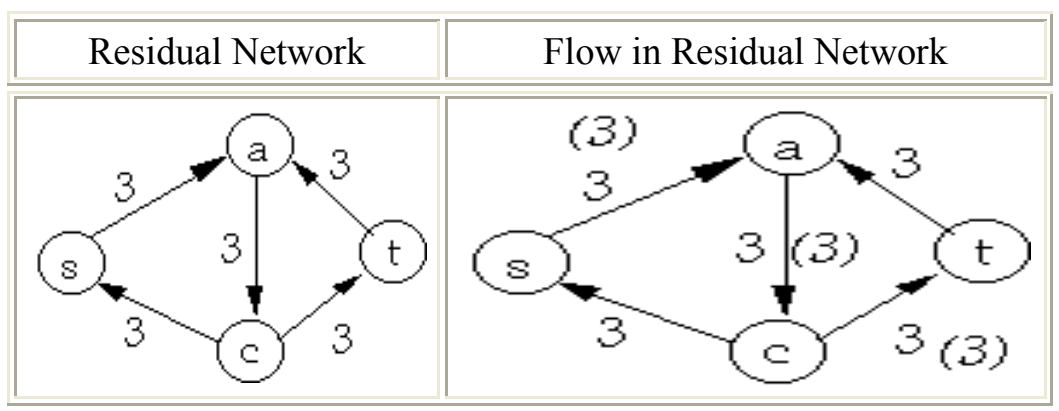

Fig. 6 


\section{Conclusions}

A network flow graph $G=(V, E)$ is a directed graph with two special vertices: the source vertex $s$, and the sink (destination) vertex $t$. Many problems in the real world or in mathematics lend themselves readily to be solved using maximum flow. "Real" networks, like the Internet or electronic circuit boards, are good examples of flow networks In case of flow of information or water or gas etc in a network the max rate of flow that is possible from one station to another can be determined. Generally graphs can be used in two situations. Firstly since graph is a very simple, convenient and natural way of representing the relationship between objects. Secondly we have graph as model solve the appropriate graph theoretic problem then interpret the solution in terms of original problem.

\section{References}

[1] G S Singh, Graph Theory, Prentice Hall of India, 2010.

[2] N Deo, Graph Theory with Applications to Engineering and Computer Science, Prentice Hall of India, 1974.

[3] S D Sharma, Operations Research, Kedarnadh and Ramnadh, 2006.

[4] J K Truss, Discrete Mathematics for Computer Scientists, Addison Wesley, 1999.

[5] D B West, Introduction to graph theory, PHI Learning, 2001.

[6] M K Das, Discrete mathematical structures for computer scientists and engineers, Narosa, 2007.

[7] Dieter van Melkebeek, Network Flow, Lecture Notes, CS 787.

[8] Richard Johnsonbaugh, Discrete mathematics, Pearson, 2007.

[9] J'L Molt, Discrete Mathematics for Computer Scientists, Prentice Hall of India, 2005

[10] Critical Path Analysis and PERT, Mind Tools.com Results. 
[11] PERT/CPM for Project Scheduling \& Management, Google search results.

[12] Jeremy Siek, Graphs and Network Flows, Lecture Notes.

[13] Combinatorial Algorithms Graphs and Network Flows, Lecture Notes, CS 660, San Diego State University. 